Cinémas

Revue d'études cinématographiques

Journal of Film Studies

\title{
Images technologiques : ce qu'il advient de la mémoire
}

\section{Yves Bédard}

Volume 1, numéro 3, printemps 1991

Nouvelles technologies : nouveaux cinémas?

URI : https://id.erudit.org/iderudit/1001068ar

DOI : https://doi.org/10.7202/1001068ar

Aller au sommaire du numéro

\section{Éditeur(s)}

Cinémas

ISSN

1181-6945 (imprimé)

1705-6500 (numérique)

Découvrir la revue

Citer cet article

Bédard, Y. (1991). Images technologiques : ce qu'il advient de la mémoire. Cinémas, 1(3), 88-101. https://doi.org/10.7202/1001068ar

\section{Résumé de l'article}

Avec l'apparition de la photographie, l'image a cessé d'être uniquement représentative, elle est devenue aussi mémoire du monde. Et le cinéma, en l'animant et en se repliant sur le réel comme son double, a fait d'elle un souvenir " vivant ". Mais il a tôt fait cependant de jouer de ses apparences et de construire des images dont le semblant de réel correspond à une mémoire non plus « objective " mais fictive, et où le monde apparaît moins comme un point de départ que comme un modèle à copier. Depuis, les développements technologiques de l'image ont accentué cette coupure : avec les images de synthèse et la télévision interactive, le spécialiste comme le spectateur moyen élaborent le spectacle personnalisé des événements qu'ils ont sous les yeux. L'écran est devenu la scène d'une mémoire du virtuel. 


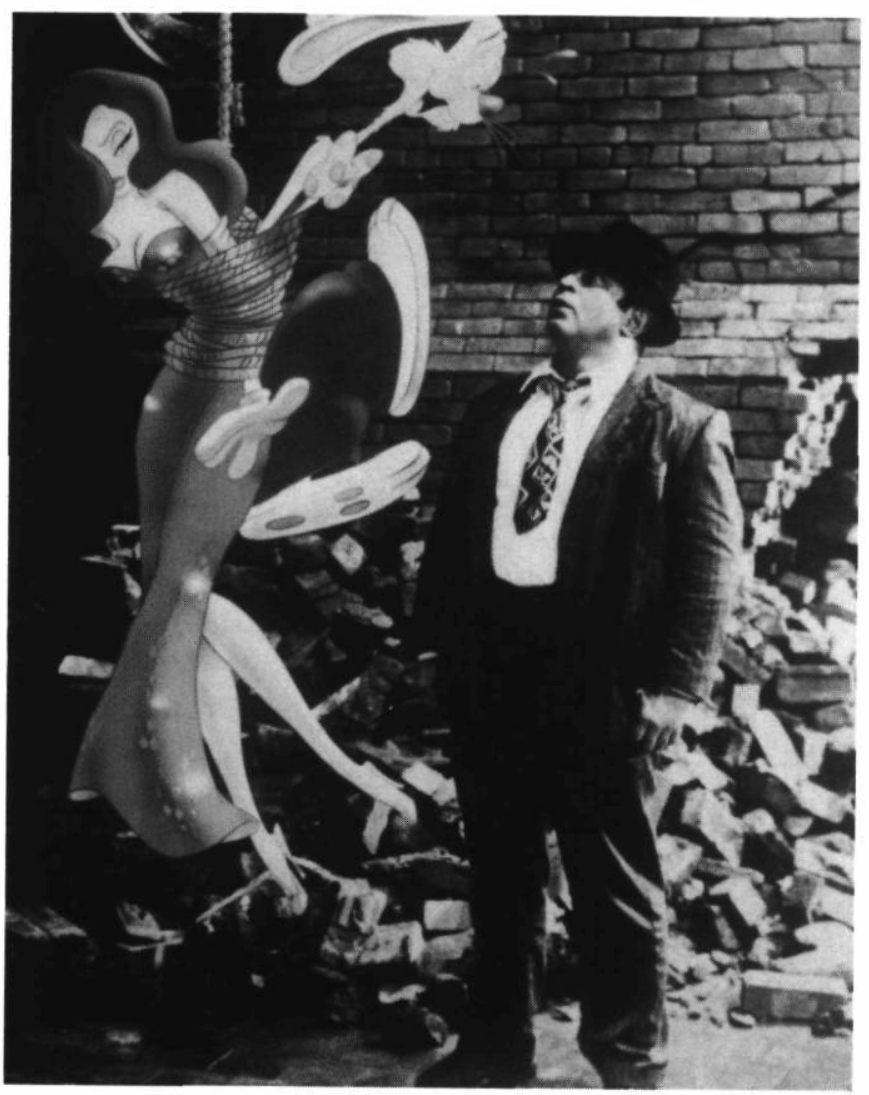

Qui a volé la peau de Roger Rabbit de Robert Zemeckis (1988)

Coll. Cinémathèque québécoise 


\title{
Images technologiques: ce qu'il advient de la mémoire
}

\section{Yves Bédard}

\begin{abstract}
RÉSUMÉ
Avec l'apparition de la photographie, l'image a cessé d'être uniquement représentative, elle est devenue aussi mémoire du monde. Et le cinéma, en l'animant et en se repliant sur le réel comme son double, a fait d'elle un souvenir "vivant». Mais il a tôt fait cependant de jouer de ses apparences et de construire des images dont le semblant de réel correspond à une mémoire non plus «objective» mais fictive, et où le monde apparaît moins comme un point de départ que comme un modèle à copier. Depuis, les développements technologiques de l'image ont accentué cette coupure: avec les images de synthèse et la télévision interactive, le spécialiste comme le spectateur moyen élaborent le spectacle personnalisé des événements qu'ils ont sous les yeux. L'écran est devenu la scène d'une mémoire du virtuel.
\end{abstract}

\begin{abstract}
With the appearance of photography, images were no longer merely representational, they became the site of the world's memory as well. Cinema, which animates photography and mirrors reality back at itself as its double, makes photography into living memory. However photography quickly finds ways to play with appearances, to construct images in which the apparent reality corresponds not to an "objective" memory but to a fiction. The real world is taken less as a point of departure, more as a model to copy from. Subsequent technological developments in imaging have accentuated this rupture. With image synthesis and interactive television, both specialists and ordinary viewers construct their own personal show out of the elements presented to them. The screen has now become the site of a memory of the virtual.
\end{abstract}


«Nous pourrons voir, mille ans après qu'elle aura jailli de la route sous le galop d'un cheval, une poussière se lever, se déployer, se dissiper, la fumée d'une cigarette se condenser puis rentrer dans l'éther.» Élie Faure Fonction du cinéma «L'image ne peut être que l'état présent d'un passé.» Jean Cayrol, Claude Durand Le Droit de regard

Au début du cinéma, il y a la suite de la photographie. Et doublement: d'abord parce que le cinéma continue la marche des technologies de l'image inaugurée par son prédécesseur, puis parce que c'est en reprenant à son compte les acquis de la photographie pour les prolonger qu'il réalise cette avancée.

\section{La vie saisie dans son cours}

La photographie, en laissant les objets se déposer en traces dans l'image, avait délivré le monde de ce qu'on pouvait appeler jusqu'alors son insaisissable déroulement. Jusque-là, en effet, toute fidèle qu'elle avait commencé d'être depuis la Renaissance, la représentation n'avait pu s'élaborer que dans une mise à distance du monde et de l'actualité des événements. Si le sujet pouvait s'inscrire dans un décor perspectiviste, sa mise en place ne survenait qu'au sacrifice de toute immédiateté. La photographie, au contraire, en absorbant directement la lumière qui se répand des corps, énonçait que les "choses avaient été», disait Barthes, qu'elles s'étaient en quelque sorte transportées elles-mêmes dans l'image et que celle-ci proposait d'elles un figement. Du même coup, un changement de nature affecta la représentation. À l'ère de la reproduction technique, elle devint fixation, rétention: le monde était là et c'est en le surprenant dans son déroulement même que l'image photographique pouvait désormais le donner à voir.

Alors que cette représentation se fondait sur l'immédiateté du rapport avec le réel, la fixité qui la caractérise venait toutefois tempérer cette poussée vers le cours évolutif de la vie en la contredisant au sein même de l'image. Celle-ci ne pouvait recueillir le monde et le tenir sous son emprise qu'en le dépouillant de son aspect dynamique. Assiégée par son immobilité, l'image photographique révélait bien le monde, mais en le pétrifiant, conservant certes des aspects du mouvement, mais l'expulsant tout autant. Et dans un siècle avide de découvertes, le mouvement apparut vite comme le manque à gagner de la nouvelle technique. C'est cette part manquante, logée dans le déborde- 
ment de la photographie, mais désignée en elle, que le cinéma injecte dans le monde représenté. Un nouvel ordre de la représentation prend alors forme, dans lequel la rétention iconique, se soudant au mouvement et faisant sienne la durée, se double d'une action protensive et épouse le destin des événements.

À quoi répond cet accomplissement si ce n'est au mythe d'immortalisation de la vie qui fonde les images photo-mécaniques? Immortaliser, dit le Petit Robert, c'est «rendre immortel dans la mémoire des hommes». Et l'apparition de la photographie, comme celle du cinéma plus tard, provoque les exclamations: pour la première fois, le réel s'infiltre automatiquement dans l'image et y laisse son empreinte pour le souvenir des générations futures. Ces techniques d'enregistrement recréent ainsi, en apparence du moins, les conditions d'une nouvelle mémoire qui arpente le monde par le regard, suppléant aux visions vagues et incertaines des images mentales qui se forment dans le souvenir, celles des images prélevées directement sur le réel. Ces instants fixés sont dès lors perçus comme autant de moments rescapés de l'oubli. Déjà, vers 1839, on dit des daguerréotypes qu'ils sont des «miroirs à mémoire», des surfaces opaques sur lesquelles non seulement les répliques du réel apparaissent, mais se maintiennent et semblent animées d'une vie propre. Benjamin cite à ce sujet Maximilian Dauthendey:

On n'osait pas au début regarder longtemps les images qu'il [Daguerre] produisait. On était intimidé par la netteté de ces hommes et on croyait que ces petits, que ces minuscules visages des personnes fixés sur la plaque étaient eux-mêmes capables de vous voir, si déconcertant était pour tout le monde l'effet produit par les premiers daguerréotypes en raison du caractère insolite de leur netteté et de leur fidélité (p. 154).

À la différence du geste pictural qui compose paysages, personnages et objets, voilà des images qui s'interposent carrément entre le monde et le souvenir humain, conservant de celui-là des traces qui deviennent matière tangible pour ce dernier.

Ayant amorcé la résurgence du passé dans le présent, la photographie avait réalisé pour l'œil un premier bouleversement symbolique des catégories du temps. Autrefois imperméables l'une à l'autre, celles-ci faisaient du regard un acte en lui-même amnésique. Certes mnémonique parce qu'il alimentait la mémoire humaine de sensations visuelles propres à édifier le souvenir, le regard était témoin du temps sans que les perceptions auxquelles il donnait lieu ne puissent être conservées de quelque façon. Mais avec son "ayant été là», la photographie faisait de l'observation un acte de rémanence. Et le cinéma, en se saisissant de la photographie pour la propulser dans la course de la 
pellicule à l'intérieur de l'appareil, prolongeait cette résurgence et lui donnait une nouvelle dimension: le passé reconquérait sa durée. En différé, il lui était dorénavant permis de retrouver et de répéter son avenir révolu. En d'autres mots, il reprenait vie.

Avec le cinéma, ce n'est donc plus tout à fait du «retour du mort» qui fut décelé dans la photographie dont il s'agit, mais à proprement parler du retour de ce qui (se) meurt. Cela n'avait pas échappé à l'attention de Bazin qui écrivait: «La photographie (...) n'a pas le pouvoir du film, elle ne peut représenter qu'un agonisant ou un condamné, non point le passage insaisissable de l'un à l'autre» (p. 69-70). Et comme en écho, Cocteau reconnaissait au cinéma la même qualité en affirmant qu'il "filme la mort au travail». Deux sortes de mémoire entraient donc en jeu pour ramener à la surface de nos vies ce qui n'existe plus: une mémoire morte et une mémoire vive.

\section{L'histoire saisie par l'image}

L'exploit du Cinématographe aura été de mettre en action un dispositif capable de se faire mémoire non pas seulement des états du monde, mais des changements qui l'animent ${ }^{1}$. Quand, un jour de 1895, un train quelconque entre en gare de La Ciotat, la chose est purement insignifiante. Il suffit pourtant qu'il rencontre une caméra sur son chemin pour passer à la postérité. Et de banale, son arrivée recréée à l'écran se mute en spectacle, devient événement et accède à l'histoire: c'est à ce moment seulement que les foules accourent et que les journaux trouvent dans la chose matière à nouvelle.

Dans cette reconquête du passé par la vision, le réel lui-même se trouve dépassé, supplanté qu'il est par sa remémoration optique. La mémoire se projette en images, s'organise en spectacle et, par ricochet, rend le réel spectaculaire. Tout, même le déjeuner d'un bébé, peut désormais défier le temps et aspirer à ce destin. Écrasé par son image, enseveli sous les signes résiduels que celle-ci a exilés de lui et conservés pour les exhiber, le monde se laisse de plus en plus appréhender par l'intermédiaire de ses traces. Les germes de la perversité qu'évoque aujourd'hui Baudrillard à propos de la relation de l'image technologique et de son référent se trouvent déjà là en train de masquer le monde au travers de ses images (p. 156).

Faire ces images revenait à agir contre le réel, même si dans l'opération celui-ci pouvait en sortir grandi. C'était détruire l'éphémère, interrompre sa disparition naturelle pour l'introduire dans la boucle de la répétition artificielle où ce qui est existentiellement unique se voit dépossédé de sa fugacité. Se 
découvrant cyclique, l'événement renonçait à son destin linéaire d'évanouissement et de mort pour entrer dans le circuit fermé d'une circularité où, capté par le regard mnémonique de la caméra, il rejaillit sous la forme du même et, bloqué dans son avenir, est condamné à repasser sans cesse par des points qu'il a déjà parcourus. Pour l'œil, désormais et littéralement, l'histoire commençait à se répéter.

Et c'est bien ce que les inventeurs du cinéma cherchaient: garder la vie en mémoire. Marey et Muybrige ne se souciaient peut-être qu'accessoirement de la synthèse du mouvement - en en privilégiant l'analyse - et n'aspiraient pas tant à le revoir qu'à mieux le voir, ils n'en étaient pas moins à une même poursuite de ses traces. Edison, on le sait, avait confié à son équipe la tâche de reproduire le mouvement pour combler son désir de rendre l'opéra éternel. Les Lumière, enfin, n'auront rêvé qu'à une seule chose: reproduire la vie pour qu'elle survienne à nouveau.

L'histoire et la remémoration des événements allaient ainsi délaisser l'ordre exclusif du langage qui leur servait jusque-là d'unique véhicule (livre, chant, parole) pour s'instrumentaliser et devenir une réalité optique. Conséquemment, la bibliothèque, où «toute la mémoire du monde» se concentre, allait voir son empire archivistique morcelé par les photothèques, cinémathèques, vidéothèques. Autour du livre s'érige ainsi l'image, nouvelle scène de l'histoire.

De l'engendrement de cette mémoire révolutionnaire, suivant en cela une loi qui ne fait qu'en démontrer l'importance, une nouvelle forme de pouvoir social allait bientôt émerger et s'imposer:

\footnotetext{
Enregistrer est depuis toujours un moyen de contrôle social, un enjeu politique, quelles que soient les techniques disponibles. Le pouvoir ne se contente plus de mettre en scène sa légitimité, il enregistre et reproduit les sociétés qu'il dirige. Stocker en mémoire, détenir l'histoire ou le temps, diffuser la parole, manipuler les informations a toujours été un des attributs des pouvoirs civils et des prêtres, depuis les Tables de la Loi (Attali, p. 173).
}

L'hégémonie de Hollywood et de son système, et puis ensuite celle des grandes agences de presse ou des gouvernements totalitaires pour les médias, allaient par la suite incarner un tel contrôle des images et de la mémoire collective à laquelle elles contribuent.

La présence de ces images, qui continuent pourtant de porter en elles des traces du monde, aura sans doute eu comme conséquence d'effacer le réel, de le dissimuler au regard sensible de la perception naturelle. Tout en rendant possibles de nouvelles vi- 
sions et la révélation d'aspects inconnus du monde, les images technologiques ont un effet de régression sur la mémoire puisque celle-ci, en passant par elles, procède d'une mise à distance du monde: «Déjà la pensée collective imposée par les divers médias visait à annihiler l'originalité des sensations, à disposer de la présence au monde des personnes en leur fournissant un stock d'informations destiné à programmer leur mémoire» (Virilio, 1980 , p. 54). Ni rapprochement complet du monde ni éloignement absolu, l'image technologique se veut les deux à la fois. Duelle, elle porte en elle des mouvements qui s'opposent par rapport au réel: médiatique, elle permet d'accéder au monde en l'oblitérant, mais en même temps, ses représentations nous en rapprochent par l'apparence de réel dont elles se revêtent.

Et si, à l'origine, le pouvoir de séduction ou d'adhésion du cinéma provient en bonne partie de l'impression de réalité qu'il dégage, il reste inséparable du caractère anamnestique de l'image. Car, imitant la perception naturelle, le cinéma donne aux images le souffle de la vie et fait revivre le réel à travers son double. C'est pourquoi un rien qui bouge, des feuilles qui s'agitent au vent, de la fumée qui s'envole d'une pipe, retiennent l'attention et suffisent au début à attirer le public. L'on sait la fortune qu'ont connue ces scènes de la vie tournées par les opérateurs de la firme Lumière. C'est que l'image filmique, en plus de naturaliser son contenu, autorise le quotidien à pointer en elle. C'est beaucoup parce qu'elle révèle ce qui, à l'œil nu, est le moins spectaculaire, le plus banal, que l'image cinématographique apparaît d'emblée comme un témoin vivant du temps:

(...) cette quotidienneté présente une structure analogue à celle du cinéma: on peut essayer de la dépasser, mais il est impossible de l'ignorer.

Grâce à elle, d'innombrables bandes de pellicule ont fixé pour l'éternité la réalité authentique des dernières décennies. Les coutumes, les changements perpétuels - et quelquefois révolutionnaires - de la civilisation, de la mode, des goûts et des aventures intellectuelles de ce siècle, survivent dans ces bobines (Biro, p. $83)$.

Aujourd'hui, saturés par les images de toutes sortes dont nous sommes journellement bombardés, insensibles devant leur opération de simple prélèvement, l'émerveillement qu'elles peuvent susciter devant le quotidien nous est devenu pour ainsi dire étranger. Mais quand l'admiration pouvait encore s'emparer des regards, quand les courtes bandes de pellicule paraissaient garantes de la postérité, la toile sur laquelle elles défilaient agissait comme un véritable écran de la mémoire. 


\section{L'image saisie par l'artifice}

Si le cinéma, et comme lui toutes les images technologiques qui l'ont suivi, enregistre le monde et s'en fait l'archive, la mémoire qu'il constitue reste purement artificielle. C'est qu'il représente moins le réel qu'il ne sécrète le sien propre. L'impression de réalité de ses représentations provient de son dispositif et nullement du monde. Celui-ci a été mis à distance en étant mis en boîte. Les aberrations du mouvement (inversions et variations de vitesse) le prouvent a contrario: quand nous voyons le mouvement battre à la mesure du réel, en harmonie avec le rythme de la vie, c'est simplement que l'œil cinématographique œuvre alors précisément à cette fin. En un sens, cet état, même intentionnel, même programmé, est accidentel. Il ne s'agit que d'une des manières par lesquelles se manifeste la perception cinématographique. C'est dire que dans le passage de la vie à l'écran, le monde, malgré l'action référentielle de l'image, s'est éclipsé.

Il n'y a pas de coextensivité de l'image cinématographique et du réel. Celui-ci reste, selon le mot de Rosset, singulier. Si le cinéma peut en projeter le double, c'est avec la distance que l'on sait. Le double cinématographique, fut-il révélateur d'aspects les plus secrets du réel, n'échappe pas à l'imperturbable singularité du réel et doit obéir aux lois de la duplication:

Car la représentation du réel se trouve ici contestée non dans sa qualité ou l'ampleur de ses performances possibles mais dans son principe même. On savait déjà que celle-ci était nécessairement limitée, imparfaite, partielle et partiale; mais voici qu'il apparaît qu'elle est de toute façon étrangère à ce qu'elle représente et comme hors de son sujet, puisque cette représentation parfaite que serait le double, réplique absolue du représenté, n'aboutit pas à une suggestion du réel mais à la relégation de celui-ci dans la nonexistence. Si le double parvient, comme il y parvient en effet de manière incomparable, à «représenter» le réel, c'est justement parce qu'il contredit toute possibilité de représentation et réussit ainsi, si l'on peut dire, la performance de présenter le réel en tant que non représentable (Rosset, p. 15-16) (c'est l'auteur qui souligne).

La duperie du double ne peut donc être que temporaire et partielle. Mais dans l'imagerie cinématographique du mouvement reproduit, l'impression de réalité continue de paraître de fois en fois, avec la même insistance, car de l'illusion et de sa conscience, la première semble toujours prête à prendre le pas sur l'autre. C'est ce qui constitue le premier effet du dispositif.

Quand Méliès métamorphose le Cinématographe en cinéma ${ }^{2}$, et quand le montage commence à organiser la représentation, cette mémoire artificielle se transforme et développe de nou- 
velles puissances. Apparitions, disparitions, surimpressions, fondus enchaînés et concaténation des plans font de l'écran le lieu d'une mémoire de l'impossible dont l'impossibilité même est déniée par sa réalisation iconique. Les espaces se fusionnent, s'enchaînent, se succèdent et créent une fiction d'événements qui n'ont de réel que leur apparence. Fiction d'événements à la croisée du réel, du virtuel, de l'imaginaire et du vraisemblable, le cinéma se détourne alors du réel pour rencontrer l'univers des fantômes, des spectres et des revenants. S'il continue de puiser ses images dans le réel, c'est pour mieux l'épuiser et plonger dans l'imaginaire pour se faire témoignage visuel de ce qui n'est pas.

Jadis matrice du temps perdu et retrouvé, décalque de la vie, l'écran devient territoire de fantasmes et, par là, s'ouvre sur les horizons de l'inexistant. Impalpable, celui-ci emprunte pour surgir les mêmes voies que le réel et s'enroule autour de la fiction comme le tourbillon autour du creux du vortex. Les images, ainsi arrachées à l'impossible, s'accrochent aux truquages et au montage et se répandent sous la forme concrète de l'ombre et de la lumière, où le vrai et le faux deviennent pour ainsi dire indistincts. Ces événements qui n'ont pas eu lieu, mais qui, même savamment truqués, ont été prélevés quelque part sur le monde, s'actualisent dans la représentation et achèvent par là une sorte de devenir-mémoire. Mémoire non plus du réel cette fois, mais de la possibilité de l'irréel, de la réalité propre de la fiction. Bref, du mythe. Mémoire fictive, pourrait-on dire enfin, qui se constitue en quelque sorte comme le souvenir d'ellemême par un renvoi à soi qui escamote le réel et lui substitue la ressemblance et surtout par la technique qui rend cette irréalité possible sur l'écran. L'impact des images sur le souvenir humain risque alors de ne plus être fonction de ce qu'elles montrent, mais de comment elles montrent:

C'est avec raison que l'intérêt de nombreux consommateurs s'attache à la technique et non aux contenus creux répétés opiniâtrement et déjà à moitié discrédités. Le pouvoir social qu'idolâtrent les spectateurs s'affirme davantage dans l'omniprésence du stéréotype imposé par la technologie que dans les idéologies vieilles et rebattues que doivent cautionner les contenus éphémères (Adorno et Horkheimer, p. 145).

Le déni du réel caché sous l'apparence de réel qui colore le plus souvent ces images pourrait porter à croire au désinvestissement de toute fonction mnémonique. Et il est vrai que des traces mnésiques optiques sont la condition d'une mémoire objective des images, même si celle-ci est condamnée à rester par- 
tielle. La valeur de preuve que l'usage a conférée aux images enregistrées en matières judiciaires est là pour en témoigner ${ }^{3}$. Mais doit-on pour autant refouler dans un non-lieu absolu de la mémoire ce qui n'aurait d'emprise que sur l'écran et prétendre que les traces qu'il contient ne viennent de nulle part? Que le souvenir expulse toute croyance, même naïve, à la fable ou au mythe? La facticité de la mémoire fictive est à considérer comme une donnée puisqu'elle définit cette mémoire dans sa distinction de la mémoire objective des images. L'imprécision, le leurre de cette mémoire fictive ne font que redoubler la fiction de la mémoire qui s'épanche en nous: de celle-ci qui fait ses films dans nos têtes, oserions-nous prétendre en l'absolue fidélité ?

Le cinéma, sous ses doubles pôles emmêlés du documentaire et de la fiction, apparaît ainsi comme une machine à effets de souvenirs pour l'œil. C'est que la fonction visualisante de l'image, comme le mirage, nous dépossède dans notre perception même de notre maîtrise sur le monde. C'est à notre esprit rationnel qu'il revient d'intervenir pour corriger ces errements optiques, distinguer l'authentique du faux, replier celui-là sur le réel et expédier celui-ci dans la fabulation. On sait combien l'aventure spectatorielle se nourrit de croyance et d'adhésion à une réalité pourtant démasquée dans son imaginaire même par la conscience. Bazin l'a bien fait observer: «Ce qu'il faut, pour la plénitude esthétique de l'entreprise, c'est que nous puissions croire à la réalité des événements en les sachant truqués» (p. 124) (c'est l'auteur qui souligne). Toutefois il nous faut bien mettre Zelig à sa place et répartir de chaque côté de la frontière du réalisé et du fictionnel les souvenirs que matérialisent les images.

$\mathrm{Si}$ la fonction originaire de l'image cinématographique fut d'offrir une scène au mimétisme de la répétition, cette fonction d'image-souvenir du réel fut vite dévoyée. L'action mémorisante du Cinématographe ayant été effectivement contredite dès Méliès. Mais cette contradiction est comme une opération de surface, l'image continuant d'œuvrer à des effets de mémoire dans un mouvement où son contenu ne cesse de transcrire la faisabilité des choses sur l'écran en même temps qu'elle l'inscrit dans un registre de l'accompli pour l'écran que nous, récepteurs de ces images, sommes tous à notre tour.

Ce jeu de caches où disparaît à la limite (c'est-à-dire dans la perfection du mirage optique et langagier du cinéma) toute habileté à discerner ne peut s'interposer totalement entre la raison et la vue. Et si, de Lumière à Méliès, nous devons constater le dépouillement de l'image de sa restrictive fonction de mémoire du monde, est-ce vraiment pour faire de Méliès le premier truqueur du cinéma? 
Avant eux [les frères Lumière], en effet, nul ne prétendait au réalisme du défilement cinématique (...). Avec Méliès et le dessinateur d'animation Émile Cohl, c'est du cinéma au-delà, au-delà de cette prétendue véracité de la lumière de la vitesse de ce film qui semble restituer l'unité sensible de la vie et qui n'est pourtant rien d'autre qu'une vision.

En deçà et au-delà des frères Lumière, le truquage n'existe pas ou n'existe plus, simplement parce que nul ne prétend à la vérité $d u$ défilé, c'est-à-dire à l'objectivité du défilement des images, il n'y a que l'illusion du trafic ou si l'on préfère, des «effets spéciaux»... (Virilio, 1984: p. 182-183) (c'est l'auteur qui souligne).

\section{La mémoire effacée}

Plongés dans le déferlement des images que domine aujourd'hui la télévision, nous voilà presque un siècle plus tard devant des images hautement perfectionnées et des techniques qui en raffinent le rendu jusqu'à l'hyperréalisme ${ }^{4}$. De Méliès à Tron ou à Qui a volé la peau de Roger Rabbit nous avons été les témoins d'une double opération de désobjectivation de l'image et d'accentuation de sa facticité. Pourtant la fine manipulation du dispositif nous donne parfois comme jamais auparavant l'impression que cette mémoire de l'impossible est plus précise, encore plus vraie que la mémoire objective de l'image. C'est que mémoire fictive et mémoire objective s'abreuvent à la même source de l'analogie, de la ressemblance, de la reconnaissance, c'est-à-dire de la confrontation de l'image avec ce que le spectateur connaît de ses propres perceptions. Nourrissez bien celle-là et il croit voir apparaître celles-ci...

Mais alors que ces images proposent du monde des représentations presque plus vraies que nature, elles s'éloignent de plus en plus des conditions de prises de vues prélevées sur le réel. Les maquettes ont perdu leur apparence de maquette et n'ont plus de maquette que le nom. Les surimpressions se multiplient de manière exponentielle à l'intérieur de l'image ou sont remplacées par autant d'incrustations qui la vident de toute proximité du monde. Et dans cette entreprise de réduction du lieu où peut se dérouler le jeu de démasquage, de recherche de la faille où l'image bascule et se dénonce comme factice, comme non-mémoire, nous sommes incités à n'y voir que du feu. Le trompel'œil de ces effets est aussi un trompe-mémoire.

Déconnectées du réel, ces images hybrides d'effets spéciaux ne disent du monde pas autre chose qu'un blanc de mémoire. Malgré leur forte impression de réalité, elles ne sont pas porteuses du monde et n'ont plus de lieu d'origine. Elles naissent d'une mémoire amnésique du réel. Mais plus encore, cette mé- 
moire du factice a commencé de s'appuyer sur une mémoire qui précède l'image. Aujourd'hui, l'ordinateur mémorise les cadrages, la place et la trajectoire des objets dans le cadre et pilote la caméra d'une prise à l'autre avant de les rassembler en un seul plan et des comédiens jouent sans décor, en faisant comme s'il y en avait. L'image, quant à elle, nous place devant ce fait accompli qui dit faussement que la chose a eu lieu, devant une sorte de mémoire qui n'a même plus à faire l'expérience du réel pour le mémoriser.

Croyant l'y retrouver, nous voyons dans ces images disparaître le réel. C'est que par le cinéma, et par les autres régimes de l'image-mouvement, le monde est réduit à sa visibilité5 et, de ce fait, ouvre la porte à l'abus de confiance. Car pour l'œil fixé sur l'écran, le visible est un état du monde où l'être et le paraître, le vrai et le faux risquent fort d'être identiques.

Avec la simulation, l'image de synthèse achève ce projet de disparition. Si le réel demeure le point de référence ultime de l'image, il n'en est pas moins totalement éclipsé de sa morphogenèse. Pour prendre forme, en effet, la figuration du monde se dispense de tout contact avec lui, elle le remplace par des modèles mathématiques et des algorithmes. Elle en anéantit toute mémoire:

La réponse donnée par l'ordinateur en temps réel n'est plus une transmission d'informations, comme le message radio ou vidéo. Elle est le résultat d'un processus de génération de sens, d'une énonciation qui ne provient pas d'un autre locuteur mais d'une machine, ou plutôt de l'hybridation de l'un et de l'autre. Sens qui ne préexiste pas, déjà enregistré dans les mémoires (comme on aime à le dire pour se rassurer), mais qui se crée au cours de la Conversation en même temps que se crée un temps nouveau (Couchot, p. 42).

Puisque la rencontre d'une caméra et de l'objet n'est plus requise, ce n'est pas l'objet mis en mémoire dans l'image qui accède à l'écran, mais ses règles d'assemblage. En ce sens, le monde s'y trouve moins représenté que mimé, et son image est moins spéculaire que spéculative ${ }^{6}$.

Dans le processus qui conduit à l'image, la simulation rejoint le monde en l'oubliant, en le liquidant ${ }^{7}$. Comme le montrent les simulateurs de vol, elle sert à ancrer dans l'homme l'expérience du réel avant même que n'ait lieu sa rencontre avec le monde, puisqu'il s'agit d'inscrire dans l'être du pilote les réflexes, les réactions et le comportement que commande sa confrontation ultérieure avec le réel. En un mot de le programmer. C'est donc dire qu'avec l'image de simulation, et en raison des conditions de sa formation, l'image technologique n'engendre pas uniquement 
une "précession des simulacres», mais aussi une précession de la mémoire. Avant la simulation, elle contenait les traces du monde et le regardeur se trouvait devant un souvenir optique. L'image se faisait mémoire dès lors qu'elle donnait à voir parce que le monde lui préexistait. Les «nouvelles images» n'ont plus besoin de lui. Il n'est que le lointain point de référence vers lequel elles convergent.

Les images technologiques ont donc altéré considérablement la dimension mémorielle dont elles étaient porteuses. Et cela vaut non seulement pour celles qui éliminent la prise de vue, mais aussi pour celles qui supposent un enregistrement. Même les images de masse peuvent maintenant être personnalisées par le Sujet qui regarde. Alors que la télévision interactive fait son entrée, le spectateur se trouve en effet clefs en main devant l'édifice d'une espèce de mémoire du virtuel où il construit sa propre représentation, unique, de l'événement qui se déroule sous ses yeux. Il y a presque dix ans déjà, Coppola décrivait les images de l'avenir: «Je m'asseois devant l'écran, je lui parle et il fait ce que je lui dis de faire.»

Des débuts de la photographie à aujourd'hui, les images technologiques n'ont cessé de se développer, se libérant des contingences de la prise de vue et du défilement prédéterminé. Pour appréhender le réel, l'ère moderne s'est d'abord donnée des images dans lesquelles se perpétue le souvenir, puis elle en a fabriquées qui atteignent le monde en le dissolvant. Contradiction? Revirement? Sans doute pas. Le régime des images technologiques concentre ainsi en lui des mouvements plus généraux de la modernité. Celle-ci, en effet, a accru les moyens d'édifier la mémoire collective, mais elle travaille en même temps à masquer les liens au passé en privilégiant l'éphémère au durable 8 .

\section{Université du Québec à Montréal et Université de Montréal}

\section{NOTES}

1 Voir, à ce sujet, la distinction entre moule photographique et modulation cinématographique dans Gilles Deleuze, Cinéma 1. L'Image-mouvement (Paris: Minuit, 1983) p. 39.

2 Voir à ce sujet Edgar Morin, Le Cinéma ou l'homme imaginaire (Paris: Gonthier, 1958) p. 43-72.

3 Depuis l'utilisation de la photographie dans les enquêtes policières jusqu'aux systèmes de surveillance vidéo ou aux saisies de rubans magnétoscopiques 
dans les locaux des stations de télévision, cette valeur, loin d'être remise en question, n'a fait que se confirmer.

${ }^{4}$ Dans une problématique embrassant tout le champ des images technologiques, il faudrait considérer les dispositifs militaires, mais aussi ceux de la médecine ou des sciences dont la relation au référent se fait sur un mode parfois très réaliste, parfois purement abstrait.

5 Voir à ce sujet Pascal Bonitzer, «L'indiscernable», "L'Ère du faux», Autrement (1986) p. 202-205.

6 Voir à ce sujet Alain Renaud, «Nouvelles images, nouvelle culture: vers un «imaginaire numérique (ou "Il faut imaginer un démiurge heureux")», «Nouvelles images, nouveau réel», Cahiers internationaux de sociologie vol. LXXXII (janvier-juin 1987) p. 131.

7 Voir à ce sujet Jean Baudrillard, «La précession des simulacres», «Le Simulacre». Traverses 10 (février 1978) p. 4.

8 Voir à ce sujet Georges Balandier, Le Désordre (Paris: Fayard, 1988) p. 244.

\section{OUVRAGES CITÉS}

Adorno, Theodor et Horkheimer, Max. «La Production industrielle de biens culturels». La dialectique de la raison. Paris: NRF Gallimard, 1974.

Attali, Jacques. Bruits. Paris: P.U.F., 1977.

Baudrillard, Jean. «Au-delà du vrai et du faux ou le malin génie de l'image». "L'Ère du faux». Autrement 76 (janvier 1986).

Bazin, André. "Mort tous les après-midi». Qu'est-ce que le cinéma, vol. 1, Ontologie et langage. Paris: Cerf, 1958.

Benjamin, Walter. «Petite histoire de la photographie». Essais I, 1922-1934. Paris: Denoël/Gonthier, 1983.

Biro, Yvette. Mythologie profane, cinéma et pensée sauvage. Paris: Lherminier, 1982.

Couchot, Edmond. "À la recherche du temps réel». "Le Jour, le temps». Traverses 35 (septembre 1985).

Rosset, Clément. L'Objet singulier. Paris: Minuit, 1985.

Virilio, Paul. Esthétique de la disparition. Paris: Balland, 1980.

Virilio, Paul. L'Horizon négatif. Paris: Galilée, 1984. 